\title{
Gamma-ray Bursts
}

\author{
Alberto J. Castro-Tirado \\ Instituto de Astrofísica de Andalucía (IAA-CSIC), P.O. Box 03004, 18080 \\ Granada, Spain; \\ ajct@iaa.es
}

\begin{abstract}
Summary. Since their discovery in 1967, Gamma-ray bursts (GRBs) have been puzzling to astrophysicists. With the advent of a new generation of $\mathrm{X}$-ray satellites in the late 90's, it was possible to carry out deep multi-wavelength observations of the counterparts associated with the long duration GRBs class just within a few hours of occurrence, thanks to the observation of the fading X-ray emission that follows the more energetic gamma-ray photons once the GRB event has ended. The fact that this emission (the afterglow) extends at longer wavelengths, led to the discovery of optical/IR/radio counterparts in 1997-2003, greatly improving our understanding of these sources. The classical, long duration GRBs, have been observed to originate at cosmological distances in a range of redshifts with 0.1685 $\leq \mathrm{z} \leq 4.50$ implying energy releases of $\sim 10^{51}$ ergs. The recent results on GRB 021004 and GRB 030329 confirm that the central engines that power these extraordinary events are due to be collapse of massive stars rather than the merging of compact objects as previously also suggested. Short GRBs still remain a mystery as no counterparts have been detected so far.
\end{abstract}

\section{Introduction}

In 1967-73 the four VELA spacecraft, that where originally designed for verifying whether the former Soviet Union abided by the Limited Nuclear Test Ban Treaty of 1963, observed 16 peculiarly strong events $[6,39]$. On the basis of arrival time differences, it was determined that they were related neither to the Earth nor to the Sun, but they were of cosmic origin. Therefore they were named cosmic Gamma-Ray Bursts (GRBs hereafter). Nearly 4000 events have been detected to date.

\section{Observational Facts and Implications}

\subsection{GRBs in the Gamma-ray Domain}

GRBs appear as brief flashes of cosmic high energy photons, carrying the bulk of their energy above $\approx 0.1 \mathrm{MeV}$ (Fig. 1). The KONUS instrument on Venera 11 and 12 gave the first indication that GRB sources were isotropically distributed in the sky $[3,44]$. Based on a much larger sample, this result was 


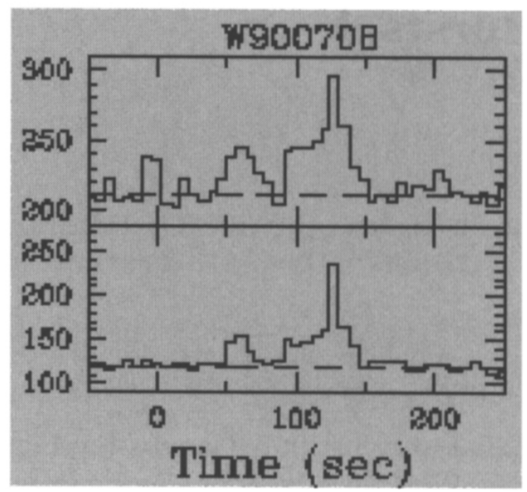

Fig. 1. One of the typical long-duration GRBs detected by WATCH on GRANAT, lasting for about $150-\mathrm{s}$ in the $8-20 \mathrm{keV}$ range (above) and in the $20-60 \mathrm{keV}$ range (below). From [42].

nicely confirmed by BATSE on board the CGRO satellite [45]. In general, there was no evidence of periodicity in the time histories of GRBs. However there was indication of a bimodal distribution of burst durations, with $\sim 25 \%$ of bursts having durations around $0.2 \mathrm{~s}$ (the short/hard GRB class) and $\sim 75 \%$ with durations around $30 \mathrm{~s}$ (the long/soft GRB class). A deficiency of weak events was noticed in the $\log N-\log S$ diagram, as the GRB distribution deviates from the $-3 / 2$ slope of the straight line expected for an homogeneous distribution of sources assuming an Euclidean geometry. However, the GRB distance scale had to remain unknown for 30 years. A comprehensive review of these observational characteristics can be seen in [22].

\subsection{Long-duration GRBs in the Electromagnetic Spectrum}

It was well known that an important clue for solving the GRB puzzle was going to be the detection of transient emission -at longer wavelengths- associated with the bursts. A review on the unsuccessful search for counterparts prior to 1997 can be seen in [10] and references therein.

\section{Solving the Distance Scale}

After the discovery of the first X-ray and optical afterglows (OA) for GRB 970228 detected by BeppoSAX [18,67], the second OA associated to a GRB was detected within the GRB 970508 error box $[5,52]$. The GRB 970508 OA light curve reached a peak in two days $(\mathrm{R}=19.7,[21])$ and was followed by a power-law decay $F \propto t^{-1.2}[11]$. Optical spectroscopy obtained during the maximum allowed a direct determination of a lower limit for the redshift of GRB 970805 ( $z \geq 0.835$ ), implying an isotropic energy release $\mathrm{E} \geq 7 \times$ $10^{51} \mathrm{erg}[47]$. It was the first proof that GRB sources lie at cosmological 
distances. The flattening of the decay in late August $1997[52,62]$ revealed the contribution of a constant brightness source -the host galaxy- that was revealed in late-time imaging obtained in 1998 [7, 12, 70]. The maximum observed 1-day after the burst has not been detected in other GRBs so far and it was interpreted by a delayed energy injection or by an axially symmetric jet surrounded by a less energetic outflow [51]. The luminosity of the galaxy is well below the knee of the galaxy luminosity function, $\mathrm{L} \approx 0.12 L^{*}$, and the detection of deep $\mathrm{Mg} \mathrm{I}$ absorption (during the bursting episode) and strong [O II] $3727 \AA$ emission (the latter mainly arising in H II regions within the host galaxy) confirmed $z=0.835$ and suggested that the host could be a normal dwarf galaxy [53], with a star formation rate (SFR) of $\sim 1.0$ $M_{\odot}$ year $^{-1}[7]$. Prompt observations at $\mathrm{cm}$ and $\mathrm{mm}$ wavelengths led to the detection of the counterpart at these wavelengths $[8,23,56]$. The fluctuations could be the result of strong scattering by the irregularities in the ionized Galactic interstellar gas, with the damping of the fluctuations with time indicating that the source expanded to a significantly larger size. However VLBI observations did not resolve the object [66]. A Fe $\mathrm{K} \alpha$ line redshifted at $z=0.835$ in the X-ray afterglow spectrum [54] was attributed to a thick torus of material surrounding the central engine [46].

About 35 host galaxies for classical, long duration GRBs, have been detected so far, in the range $0.1685 \leq \mathrm{z} \leq 4.50$ (if ESO 184-G82 is excluded). None of the hosts are brighter than the knee of the Iuminosity function $L^{*}$ at their redshift, but the GRB hosts are noticeable bluer than typical galaxies of similar magnitude [63].

\section{The Existence of Collimated Emission}

GRB 990123 was the first event for which contemporaneous optical emission was found simultaneous to the gamma-ray burst, reaching $V \sim 9$ [1]. This optical flash did not track the gamma-rays and did not fit the extrapolation of the BeppoSAX and BATSE spectra towards longer wavelengths. This optical emission was interpreted as the signature of a reverse shock moving into the ejecta [60]. A brief radio transient was also detected [24] coincident with the optical counterpart [49] and spectroscopy indicated $z=1.599$ [2]. A break observed in the light curve $\sim 1.5$ days after the high energy event suggested the presence of a beamed outflow $[13,25,41]$, thus reducing the energy release by $\sim 10^{2}$. A weak magnetic field in the forward shock region could account for the observed multiwavelength spectrum in contrast to the high-field magnetic field for GRB 970508 and it seems that the emission from the three regions was first seen in this event [28]: the internal, reverse and forward shocks.

Further support for a jet-like outflow came for GRB 990510, the first burst for which polarized optical emission was detected $(\Pi=1.7 \pm 0.2 \%$, $[19,68])$. This confirmed the synchrotron origin of the blast wave itself and represented another case for a jet-like outflow [64], as has been later seen in other events. 


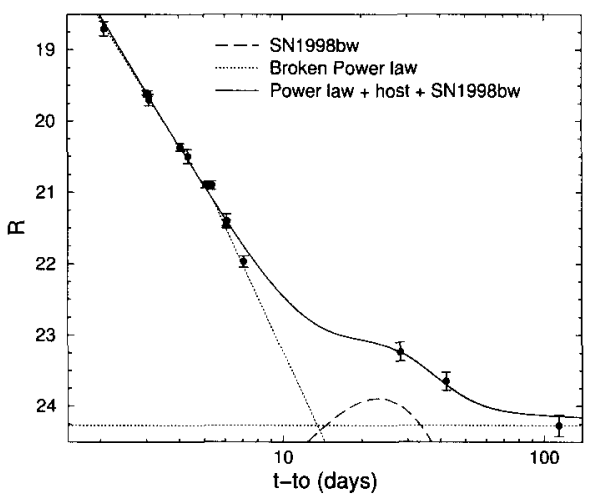

Fig. 2. The GRB 991208 R-band light curve (solid line) fitted with a SN1998 bwlike component at $z=0.706$ (long dashed line) superposed to the broken power-law OA light curve displaying the second break at $\mathrm{t}_{\text {break }} \sim 5 d$ (with $\alpha_{1}=-2.3$ and $\alpha_{2}$ $=-3.2$, short dotted lines) and the constant contribution of the host galaxy $(\mathrm{R}=$ $24.27 \pm 0.15$, dotted line). From [15].

\section{Dark GRBs}

Following the unsuccessful search for an OA for GRB 970111 [9], intensive work was conducted for GRB 000210, a burst that was followed up by CHAN$D R A$. The fact that no OA was detected despite of deep optical searches down to $\mathrm{R} \sim 23.5$ implied to classify the event as a another "dark" GRB. A constant brightness optical counterpart $(\mathrm{R}=23.5,[32])$ was coincident with the $1.6^{\prime \prime}$ error box derived by CHANDRA [30] implying that this was the likely host galaxy. A radio transient was discovered with the VLA and from the $\mathrm{X}$-ray spectrum, it was derived that either the gas is local to the GRB or that the gas is located in a dusty, gas-rich region of the galaxy [55], which is observed to harbor considering star formation [33].

In at least another three cases (GRB 981226, GRB 990506 and GRB 001109), radio transients were detected without accompanying optical/IR transients. The observed fraction of dark GRBs detected so far is $\sim 40 \%$. This could be due to intrinsic faintness because of a low density medium, high absorption in a dusty environment, or Lyman limit absorption in high redshift galaxies $(z>7)$. If GRBs are tightly related to star-formation, a substantial fraction of them should occur in highly obscured regions. For instance, most of star formation in the Hubble Deep Field is so enshrouded by dust that starlight from the galaxies detected by SCUBA is attenuated by a factor of $\sim 10^{2}[38]$.

\section{The Detection of X-ray Lines}

The GRB 011211 (at $\mathrm{z}=2.14$ ), displayed rapid variations in the $\mathrm{R}$-band light curve approximately 0.5 days after the burst, suggesting that they were 


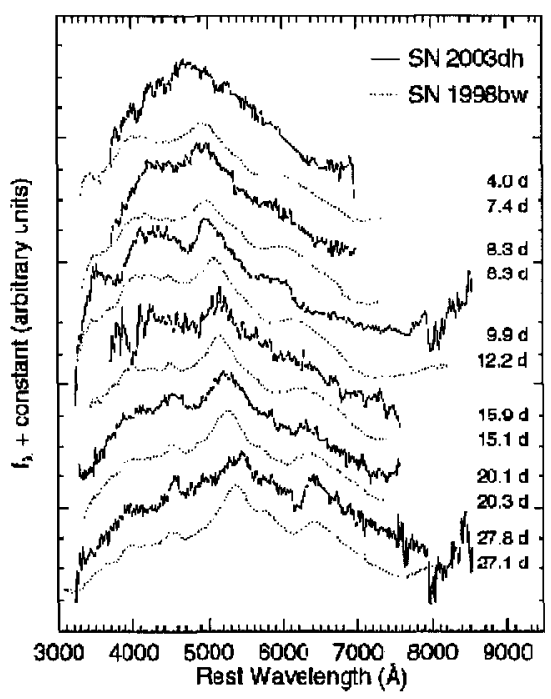

Fig. 3. Spectral evolution of the combined GRB 030329, the associated SN 2003dh and its host galaxy (no reddening correction applied). The lower spectra, dominated by SN $2003 \mathrm{dh}$, reveals the typical broad-band SN signatures. To ease comparison, the spectrum of SN $1998 \mathrm{bw}$ at 33 days after the explosion is shown at the GRB 030329 redshift. From [36].

due to density fluctuations near the central engine on spatial scales of approximately 40-125 AU [37]. The early afterglow spectrum was obtained by $X M M-N e w t o n$, which observed 11 hours after the initial burst, and appeared to reveal decaying $\mathrm{H}$-like $\mathrm{K}$ emission lines of $\mathrm{Mg}, \mathrm{Si}, \mathrm{S}, \mathrm{Ar}$ and $\mathrm{Ca}$, arising in enriched material with an outflow velocity of order $\sim 0.1 \mathrm{c}[57]$. This was attributed to matter ejected from a massive stellar progenitor occurring shortly before the burst itself. Thermal emission, from an optically thin plasma, is the most plausible model that could account for the soft X-ray emission. The X-ray spectrum evolved with time over the first $12 \mathrm{ksec}$, suggesting that thermal emission dominated the early afterglow spectrum, whilst a powerlaw component dominated the latter stages. This implies that the progenitor of the GRB was a massive star and that the mass of the ejected material in GRB 011211 was estimated to be $\sim 4-20 M_{\odot}[58]$.

\section{The GRB-SN Connection}

A peculiar Type Ic supernova (SN 1998bw) was found in the error box for the soft GRB 980425 by Galama et al. [27]. The SN lies was associated to the galaxy ESO 184-G82, an actively star forming SBc sub-luminous galaxy at $z=0.0085$. The fact that the SN event occurred within \pm 1 day of the GRB event, together with the relativistic expansion speed derived from the 
The total energy released would be $8 \times 10^{47} \mathrm{erg}$ which is about $\sim 10^{5}$ smaller than derived for "classical" GRBs. Follow-up HST observations of ESO 184G82 2.1 yr after the event, revealed an object consistent with being a point source within the astrometric uncertainty of $0.018^{\prime \prime}$ of the SN position. The object is located inside a star- forming region and is at least one magnitude brighter than expected for the $\mathrm{SN}$ based on a simple radioactive decay model, implying either a significant flattening of the light curve or a contribution from an underlying star cluster [26].

Independently, Castro-Tirado and Gorosabel [14] and Bloom et al. [4] also suggested the presence of an underlying SN in GRB 980326. Reichart [59] also proposed that a type Ib/c supernova lay "behind" another GRB (GRB 970228), overtaking the light curve two weeks after. This fact seems to be confirmed by a following work [29]. Further evidences have been found for another bursts [15, 20, 31, 34, 43]. See Fig. 2.

For GRB 021004, at least seven absorption line complexes spanning a velocity range of about $3000 \mathrm{~km} / \mathrm{s}$ were found in the vicinity of the host galaxy, at $\mathrm{z}=2.33[16]$. This observational evidence was interpreted as the presence of a multiple shell structure formed by the winds around the massive star progenitor, which it is thought to have been a Wolf-Rayet star [16, 48,61].

GRB 030329 is the nearest classical, long duration GRB detected so far, at $\mathrm{z}=0.1685$. Due to its proximity, spectroscopic campaigns started already 1 day after the event, which led to the detection of SN absorption features superposed on the power-law OA spectrum. These signatures increased with time and resembled remarkably those of the SN1998bw/GRB 980425 spectrum. Thus, in spite of no optical bump being present in the optical/NIR light curve [35], the underlying object was dubbed SN2003dh (type Ic) [65] and from VLT spectroscopy, a photospheric expansion velocity of $\sim 0$.1c one week after the GRB was derived [36], comparable to the value found for SN1998bw. This event is therefore considered as the smoking gun that has allowed to definitively connect the collapse of massive stars with the longduration GRBs, as was already proposed in 1974 [17] amongst the more than 100 theoretical models for explaining their origin.

It is possible then, in these powerful supernovae also dubbed "hypernovae" [50], that the explosions will be asymmetric and we are observing the GRB produced by a relativistic jet propagating along the rotation axis of the massive progenitor [69].

Acknowledgement. I am very grateful to M. E. Alcoholado-Feltström for her continuous support. This work has been partially financed by the Spanish Ministerio de Ciencia y Tecnología research project PNAYA 2002-0802, which makes use of the EU FEDER funds. 


\section{References}

1. C. Akerloff et al. : Nature 398, 400 (1999)

2. M. Andersen et al. : Science 283, 2075 (1999)

3. J.-L. Atteia et al. : Astrophys. J. Suppl. 64, 305 (1987)

4. J.S. Bloom et al. : Nature 401, 453 (1999)

5. H. Bond: IAUC 6655 (1997)

6. J.T. Bonnell, R.W. Klebesadel: In: Gamma-Ray Bursts, 3rd Huntsville Workshop. ed. C. Kouveliotou et al. (AIP 384, 1996) p. 977

7. J.S. Bloom et al. : Astrophys. J. Lett. 507, L25 (1998)

8. M. Bremer et al. : Astron. Astrophys. 332, L13 (1998)

9. A.J. Castro-Tirado et al. : IAUC 6598 (1997)

10. A.J. Castro-Tirado: In: Ultraviolet Astrophysics: Beyond the IUE Final Archive. ed. R. Gonzalez-Riestra et al. (ESA Conf. Proc. SP-413, 1998) p. 659.

11. A.J. Castro-Tirado et al. : Science 279, 1011 (1998)

12. A.J. Castro-Tirado et al. : IAUC 6848 (1998)

13. A.J. Castro-Tirado et al. : Science 283, 2069 (1999)

14. A.J. Castro-Tirado, J. Gorosabel: Astron. Astrophys. Suppl. 138, 449 (1999)

15. A.J. Castro-Tirado et al. : Astron. Astrophys. 370, 398 (2001)

16. A.J. Castro-Tirado et al. : In preparation

17. S.A. Colgate: Astrophys. J. 187, 333 (1974)

18. E. Costa et al. : Nature 387, 783 (1997)

19. S. Covino et al. : Astron. Astrophys. 348, L1 (1999)

20. M. Della Valle et al. : Astron. Astrophys. 406, L33 (2003)

21. S.G. Djorgovski et al. : Nature 387, 876 (1997)

22. G.J. Fishman, C.A. Meegan: Ann. Rev. Astron. Astrophys. 33, 415 (1995)

23. D.A. Frail et al. : Nature 389, 261 (1997)

24. D. Frail et al. : GCN 211 (1999)

25. A. Fruchter et al. : Astrophys. J. Lett. 519, L13 (1999)

26. J.P. Fynbo et al. : Astrophys. J. Lett. 542, L89 (2000)

27. T. Galama et al. : Nature 395, 670 (1998)

28. T. Galama et al. : Nature 398, 394 (1999)

29. T. Galama et al. : Astrophys. J. 536, 185 (2000)

30. M. Garcia et al. : GCN 548 (2000)

31. P.M. Garnavich et al. : Astrophys. J. 582, 924 (2003)

32. J. Gorosabel et al. : GCN 783 (2000)

33. J. Gorosabel et al. : Astron. Astrophys. 400, 127 (2003)

34. J. Greiner et al. : Astrophys. J., in press

35. S. Guziy et al. : In preparation

36. J. Hjorth et al. : Nature 423, 847 (2003)

37. S.T. Holland et al. : Astron. J. 124, 639 (2002)

38. D.H. Hughes, J.S. and Dunlop: In: Highly Redshifted Radio Lines. eds. C.L. Carilli et al. (ASP Conf. Series 156, 1999) p. 99

39. R. Klebesadel, I. Strong, R. Olson: Astrophys. J. Lett. 182, L85 (1973)

40. S. Kulkarni et al. : Nature 395, 663 (1998)

41. S. Kulkarni et al. : Nature 398, 389 (1999)

42. N. Lund, S. Brandt, A.J. Castro-Tirado: In: Gamma-Ray Bursts, 1st Huntsville Workshop. eds. G.J. Fishman et al. (1992) p. 53

43. N. Masetti et al. : Astron. Astrophys. 404, 465 (2003) 
44. E.P. Mazets et al. : Astrophys. Space Sci. 80, 3 (1981)

45. C.A. Meegan et al. : Nature 355, 143 (1992)

46. P. Mészáros, M.J. Rees: Mon. Not. R. Astron. Soc. 299, L10 (1998)

47. M.R. Metzger et al. : Nature 387, 878 (1997)

48. N. Mirabal et al. : astro-ph 0303616 (2003)

49. S.C. Odewahn et al. : GCN 201 (1999)

50. B. Paczyński: Astrophys. J. Lett. 494, L45 (1998)

51. A. Panaitescu, P. Mészáros, M.J. Rees: Astrophys. J. Lett. 503, L314 (1998)

52. H. Pedersen et al. : Astrophys. J. 496, 311 (1998)

53. E. Pian et al. : Astrophys. J. Lett. 492, L103 (1998)

54. L. Piro et al. : Astrophys. J. Lett. 514, L73 (1999)

55. L. Piro et al. : Astrophys. J. 577, 680 (2002)

56. G. Pooley, D. Green: IAUC 6670 (1997)

57. J.N. Reeves et al. : Nature 416, 512 (2002)

58. J.N. Reeves et al. : Astron. Astrophys. 403, 463 (2003)

59. D. Reichart: Astrophys. J. Lett. 521, L111 (1999)

60. R. Sari, T. Piran: Astrophys. J. Lett. 520, L641 (1999)

61. B.E. Schaefer et al. : Astrophys. J. 588, 387 (2003)

62. V.V. Sokolov et al. : Astron. Astrophys. 334, 117 (1998)

63. V.V. Sokolov et al. : Astron. Astrophys. 372, 438 (2001)

64. K.Z. Stanek et al. : Astrophys. J. Lett. 522, L39 (1999)

65. K.Z. Stanek et al. : Astrophys. J. Lett. 591, L17 (2003)

66. G.B. Taylor et al. : Nature 389, 263 (1997)

67. J. van Paradijs et al. : Nature 386, 686 (1997)

68. R.A.M.J. Wijers et al. : Astrophys. J. Lett. 523, L33 (1999)

69. S.E. Woosley: Astrophys. J. 405, 273 (1993)

70. S.V. Zharikov, V.V. Sokolov, Yu.V. Barishev: Astron. Astrophys. 337, $356(1998)$ 